\title{
Evaluating the microscopic effect of brushing stone tools as a cleaning procedure
}

\author{
Antonella Pedergnana ${ }^{a, *}$, Ivan Calandra ${ }^{a}$, Konstantin Bob ${ }^{\mathrm{b}}$, Walter Gneisinger ${ }^{\mathrm{a}}$, \\ Eduardo Paixão ${ }^{\text {a,c,d }}$, Lisa Schunk ${ }^{\text {a,c }}$, Andreas Hildebrandt ${ }^{\text {b }}$, João Marreiros ${ }^{\text {a, c, d }}$ \\ ${ }^{a}$ TraCEr, Laboratory for Traceology and Controlled Experiments at MONREPOS Archaeological Research Centre and Museum for Human Behavioural Evolution, RGZM, \\ Neuwied, Germany \\ b Scientific Computing and Bioinformatics, Institute of Computer Science, Johannes Gutenberg University, Staudingerweg 9, 55128, Mainz, Germany \\ ${ }^{\mathrm{c}}$ Institute for Prehistoric and Protohistoric Archaeology, Johannes Gutenberg University, Schönborner Hof, Schillerstraße 11, 55116, Mainz, Germany \\ d ICArEHB - Interdisciplinary Center for Archaeology and Evolution of Human Behaviour, University of Algarve, Faro, Portugal
}

\section{A R T I C L E I N F O}

\section{Keywords:}

Cleaning protocols

Brushing

Stone tools

Use-wear analysis

Confocal microscopy

\begin{abstract}
A B S T R A C T
Cleaning stone tool surfaces is a common procedure in lithic studies. The first step widely applied at any archeological site (and/or at field laboratories) is the gross removal of sediment from the surfaces of artifacts. Lithic surface alterations due to mechanical action applied in wet or dry cleaning regimes have never been examined at a microscopic scale. This could have important implications in traceology, as any modern surface modifications inflicted on archeological artifacts might compromise their functional interpretations. The current trend toward quantification of use-wear traces makes the testing even more important, as even slight, apparently invisible surface alterations might be measured.

In order to evaluate the impact of common cleaning procedures, we undertook a controlled experiment. The main aim of this experiment was to assess the effects that brushing actions applied for removing sediment particles have on flint and quartzite surfaces.

All surfaces were analyzed with confocal microscopy before and after having been brushed to quantify possible changes in the micro-topography. Surface roughness parameters (ISO 25178-2 among others) were applied.

Nine parameters changed significantly when mechanical actions were applied to lithic surfaces, meaning that some changes in the surface micro-topography were detected. Therefore, archeologists need to be cautious when applying prolonged mechanical actions for cleaning archeological stone tools.
\end{abstract}

\section{Introduction}

Cleaning stone tool surfaces prior to microscopic observation is a necessary step in any microscopic analysis. This is done to remove any undesired substance (e.g. contaminants, sediment concretions, ink and varnish) which would cover use-wear features. Artifacts are also cleaned prior to other kinds of analysis, such as technological and refitting studies. Particularly, sediment concretions have to be removed to allow analysts to carefully inspect the technological traits of knapped tools (e. g. ripples, negatives of previous removals).

Depending on the aim of the study, raw materials, personal preference or experience, different protocols are adopted in different laboratories, but these are rarely adequately described in publications. The main consequence of this disparity of applied procedures is that results are hardly comparable and not reproducible. Since the possible impact that cleaning protocols and the handling of objects might have on functional analysis itself also seems to be underestimated, the effects of chemical products and mechanical actions involved in cleaning protocols commonly applied to archeological material before use-wear analysis should be tested (Macdonald and Evans, 2014). For instance, the use of acidic solutions (commonly hydrochloric acid diluted to different concentrations) can cause patination of certain chert varieties (Éva Halbrucker, pers. comm.). The use of basic solutions (for instance sodium hydroxide $\mathrm{NaOH}$, or potassium hydroxide $\mathrm{KOH}$ ) to neutralize acid residues is still very debatable. In fact, if done in an unsystematic way, this could lead to the deposition of salts on the surfaces and in the pores of stone tools. Certain salts could, for example, damage rocks by volume changes during crystallization (Yu and Oguchi, 2009).

\footnotetext{
* Corresponding author.

E-mail addresses: antonella.pedergnana@gmail.com, pedergnana@rgzm.de (A. Pedergnana).
} 
In this paper, we test the first step generally applied in any cleaning protocol: the mechanical removal of sediment particles (if present) and its potential effect on the surface of the artifacts. As this is very frequently done either by brushing the tools' surfaces or by gently rubbing them with one's fingers, we decided to test these two approaches separately. While soft brushing can be performed under both dry and wet conditions, finger-rubbing is done with the addition of water in order to soften the sediment, therefore facilitating its quick removal.

Soft brushing is applied in many cleaning protocols - both on-site and in laboratories - as it is considered to be relatively harmless (e.g. Evans and Donahue, 2005; Hamon and Plisson, 2008; Evans and MacDonald, 2011), even if caution has been raised by some analysts (e.g. Rots, 2010, p. 46). The main issue in applying a variable range of pressures with a relatively soft material (plastic filaments installed in brush heads) onto lithic surfaces covered in sediment is the possibility of creating "modern wear". If all surface modifications are to be explained in terms of tribological relationships between two or more bodies, the effect that toothbrushes have on stone tools during common cleaning practice at archeological sites/laboratories must be tested.

One could argue that, since contact between brushes/fingers and lithic tools is not prolonged, no particular surface changes should occur. The detection of such changes likely depends on the scale of analysis. Surface modification from brushing invisible at low magnifications may only become visible at a much finer scale (nanometers). This would mean that quantifying the brushed surfaces might detect changes invisible to the naked eye and under low power microscopes. Moreover, we should not forget that the sediment particles removed during this practice may play a crucial role. When sedimentary concretions are rubbed or brushed, a certain pressure is applied, causing single sediment particles (such as quartz grains) to be dragged across the tools' surfaces. These particles are considered to be responsible for the formation of linear features (e.g. striations) during use (Brink, 1978; Kamminga, 1979; Mansur-Franchomme, 1983; Pedergnana, 2017), and are therefore fundamental to the reconstruction of the kinematics of tool use. Hence, the formation of modern striations, unrelated to use, should be avoided to prevent compromising the functional interpretations of archeological objects. The production of "modern traces" would add obstacles to the daily routine of use-wear analysts. Knowing the difficulty of distinguishing use-wear from post-depositional surface modifications (PDSMs) (Levi Sala, 1986; Werner, 2018), any additional cause of equifinality of surface modifications is obviously unwanted. On the other hand, if brushing is capable of altering the surfaces of stone tools, it should be acknowledged in order to promptly act by modifying the selected cleaning protocols in archeological and conservation projects.

To understand the implications of the above-presented scenario, we undertook an experiment to test the effects of both brushing and rubbing actions on flint and quartzite surfaces (fine and coarse-grained raw materials respectively) to remove sediment particles. The surfaces of the experimental tools were analyzed with a laser-scanning confocal microscope (LSCM) before and after cleaning. A number of surface parameters (among others, ISO 25178-2; International Organization for Standardization, 2012) were used for comparing the surface textures of the experimental flakes.

\section{Materials and methods}

\subsection{Samples}

Four quartzite and four flint flakes $(\mathrm{N}=8)$ were knapped from two blocks. Two different raw materials were selected to assess whether they are similarly affected by mechanical actions or not. Only one core per raw material was used in order to limit raw material intra-variability. The flint was collected in a quarry in Harmignies, Belgium, while the quartzite cobble was collected in a quarry of deposits from the river Rhine in Heimbach-Weiß/Neuwied (Rhineland-Palatinate, Germany).
A system of three beads was applied on all samples in order to be able to locate the same areas before and after the experiments (as described in Calandra et al., 2019a). Two samples (one per raw material) were kept as controls and were simply cleaned and measured twice. Two samples (one per raw material) were meant to mimic sediment removal via brushing. Two others were meant to test brushing alone (without the application of sediment). Sediment applied to the remaining two samples (one per raw material) was not removed through brushing. Soft removal with tap water and hand rubbing was applied (Table 1).

\subsection{Experimental procedure workflow}

The surfaces of the tools were analyzed before and after brushing/ rubbing by using a sample coordinate system in conjunction with a laserscanning confocal microscope. The workflow applied followed several steps:

1. Apply beads to define the coordinate system on the samples (Calandra et al., 2019a);

2. Clean the samples;

3. Acquire surface measurements (confocal microscope) on two locations (Area 1 and Area 2) per sample (Fig. 2);

4. Perform experiments (Fig. 1);

4a Apply standard sediment to two flint (FLT3-10 and 13) and two quartzite (QTZ3-3 and 13) samples;

4b Brush (FLT3-10 and QTZ3-13) or gently rub off (FLT3-13 and QZT3-3) the sediment;

5. Clean the samples;

6. Acquire surface measurements (confocal microscope) of the same locations acquired previously;

7. Process the surface data acquired.

\subsubsection{Cleaning procedure}

The samples were cleaned twice: (1) before the first set of measurements, i.e. before the application of the standard sediment, and (2) after the conclusion of the experiment (after brushing/rubbing) but before the second set of data acquisition.

The cleaning protocol comprised several steps:

1. Rinse under tap water;

2. Ultrasonic bath in detergent solution ( $1 \mathrm{~g} / \mathrm{L}$ - Plurafac LF 901 in distilled water) for $15 \mathrm{~min}$ at $40{ }^{\circ} \mathrm{C}$;

3. Rinse under tap water to remove the detergent solution;

Table 1

The experimental samples and applied actions. FLT3 and QZT3 designate the two raw material blocks (flint and quartzite respectively), from which the experimental flakes were knapped. A control sample is present for each raw material (no sediment and no brushing).

\begin{tabular}{|c|c|c|c|c|}
\hline Sample & $\begin{array}{l}\text { Raw } \\
\text { material }\end{array}$ & $\begin{array}{l}\text { Application of } \\
\text { sediment }\end{array}$ & $\begin{array}{l}\text { Mechanical } \\
\text { action }\end{array}$ & $\begin{array}{l}\text { Nomenclature } \\
\text { used }\end{array}$ \\
\hline FLT3-8 & Flint & \multicolumn{2}{|c|}{$\begin{array}{c}\text { Control sample: no treatment } \\
\text { applied }\end{array}$} & Control \\
\hline FLT3-9 & Flint & $\begin{array}{l}\text { No sediment } \\
\text { applied }\end{array}$ & Brushing & BrushNoDirt \\
\hline FLT3-13 & Flint & $\begin{array}{l}\text { Application of } \\
\text { sediment }\end{array}$ & Rubbing & RubDirt \\
\hline FLT3-10 & Flint & $\begin{array}{l}\text { Application of } \\
\text { sediment }\end{array}$ & Brushing & BrushDirt \\
\hline QTZ3-2 & Quartzite & \multicolumn{2}{|c|}{$\begin{array}{c}\text { Control sample: no treatment } \\
\text { applied }\end{array}$} & Control \\
\hline QTZ3-5 & Quartzite & $\begin{array}{l}\text { No sediment } \\
\text { applied }\end{array}$ & Brushing & BrushNoDirt \\
\hline QTZ3-3 & Quartzite & $\begin{array}{l}\text { Application of } \\
\text { sediment }\end{array}$ & Rubbing & RubDirt \\
\hline QTZ3-13 & Quartzite & $\begin{array}{l}\text { Application of } \\
\text { sediment }\end{array}$ & Brushing & BrushDirt \\
\hline
\end{tabular}




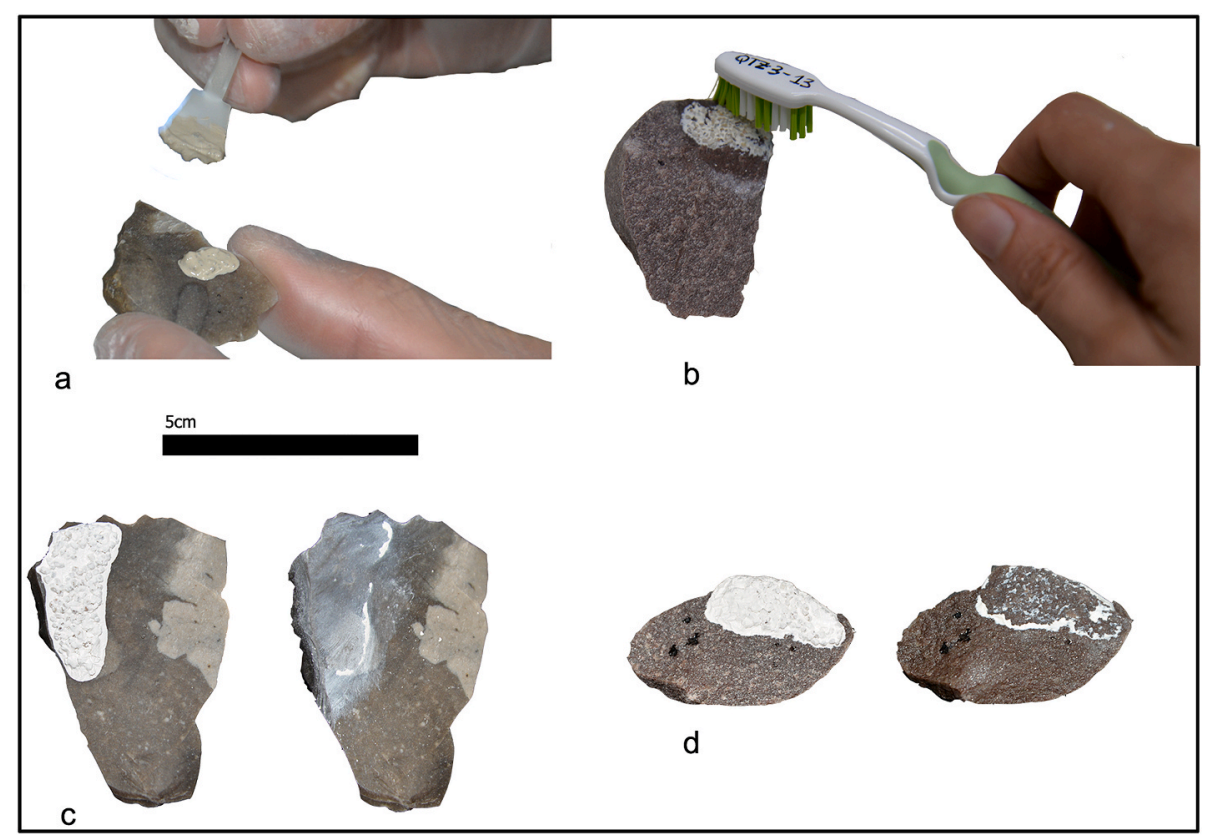

Fig. 1. Illustration of the experiment. a) Application of standardized sediment on a flint flake; b) Mechanical brushing of the dry sediment from the surface of a quartzite flake (QTZ3-13); c) Before and after the standard sediment was removed through brushing on a flint flake (FLT3-10); d) Before and after soft removal of the sediment (water cleansing) on a quartzite flake (QTZ3-3).

4. Ultrasonic bath in distilled water for $5 \mathrm{~min}$ at $40^{\circ} \mathrm{C}$;

5. Application of acetone (technical grade) on the area to be measured prior to analysis with confocal microscopy.

After the experiment, an additional step was added to the above cleaning procedure between steps 4 and 5 . A variable volume of hydrochloric acid solution $(\mathrm{HCl}$ diluted to $10 \% \mathrm{v} / \mathrm{v}$ ) was pipetted onto the areas where the sediment was previously present to remove all persistent sediment residues. The same treatment was applied to the control samples. After $4 \mathrm{~min}$, acid residues were removed through a bath in $1 \mathrm{~L}$ of tap water. Afterward, all surfaces were rinsed with distilled water.

\subsubsection{Experiments}

In order to mimic sediment, a "standard sediment mixture" was prepared using 1:2:1 parts (weight) of calcium hydroxide $\left(\mathrm{Ca}(\mathrm{OH})_{2}\right)$ (i.e. hydrated lime) (Kremer Pigmente GmbH \& Co. KG), sieved quartz grains and water. The quartz grains used ranged from 0.7 to $1.25 \mathrm{~mm}$ in diameter and were semi-angular, i.e. from a natural deposit and not machine-crushed. The mixture was mechanically homogenized and $0.5 \mathrm{~g} \pm 10 \%$ was applied with a soft plastic spatula onto previously measured flint and quartzite surfaces (Fig. 1: a). The mixture was applied to four samples (two quartzite and two flint flakes). The mixture was left to air-dry (converting to mostly $\mathrm{CaCO}_{3}$ via reaction with $\mathrm{CO}_{2}$ ) for 12 days. When the mixture was sufficiently solidified to mimic a calcium carbonate crust, the sediment was mechanically removed from two samples (one flint and one quartzite) using a hard toothbrush (the hardest grade available) wetted with tap water (Fig. 1: b). All remnants of the sediment mixture were macroscopically removed. The time required for manual brush cleaning ranges from 120 to 130 s per sample. On the other two samples on which dirt was applied but not removed through brushing, the mixture was gently removed by hand rubbing after ca. $60 \mathrm{~s}$ baths in tap water. On the two samples where the mixture was not applied and only dry brushing was performed, the duration of the action was the same as for the samples with dirt (i.e. 120 s).

\subsection{Microscopy}

Digital microscopy (ZEISS Smartzoom5) was used to document the position of the beads composing the coordinate system and to rapidly screen the surfaces of the flakes after being brushed. This was done in order to document possible wear traces formed during the removal of the sediment mixture.

A confocal microscope (LSM 800 MAT mounted onto an Axio Imager. Z2 Vario light microscope, Carl Zeiss Microscopy $\mathrm{GmbH}$ ) was used to measure two randomly selected areas per sample before $(n=16)$ and after $(n=16)$ the experiments (Fig. 2: a, c, e, g).

When the sediment mixture was applied, the two scans were acquired on the area that was previously covered by sediment. On the other samples, the scans were acquired on randomly selected areas, all relatively far from the edge.

Hence, 32 measurements were performed in total. The C EpiplanApochromat $50 \times / 0.95$ objective was used for all data acquisitions. The field of view (FOV) was $255.56 \times 255.56 \mu \mathrm{m}$ and the pixel size (spacing) was $0.0852 \mu \mathrm{m}$. The acquisitions were performed with the settings given in Supplementary Material (SupplMat1). The image stack was processed without any noise cut (i.e. all data points are considered) and exported into two surfaces (topography and maximum intensity) in SUR format for further processing (section 2.4). In addition to the surface textural data, extended depth of focus (EDF) bright field microscopic images of all areas of interests were acquired with the same objective by stitching $2 \times 2$ tiles covering a total area of $268.48 \times$ $268.48 \mu \mathrm{m}$ (Fig. 2 : b, d, f, h). The step size was set to $1 \mu \mathrm{m}$ for EDF. Note that due to the stitching process, it is common that the resulting images have a size different than the acquisition size.

One area of one sample (FLT3-8 Area 1) was not correctly acquired before the cleaning experiment but this was noticed only after the experiment had been performed. Since there was no baseline for this area to compare to, it was excluded from the subsequent analyses. Therefore, 30 measurements were analyzed. Note that the second area of this sample was acquired correctly, meaning that all samples were included in all further analyses.

\subsection{Analysis workflow for the processing of surface data}

The workflow for surface data processing has been adapted from Arman et al. (2016) and Calandra et al. (2019b). It followed several steps 


\section{FLINT}
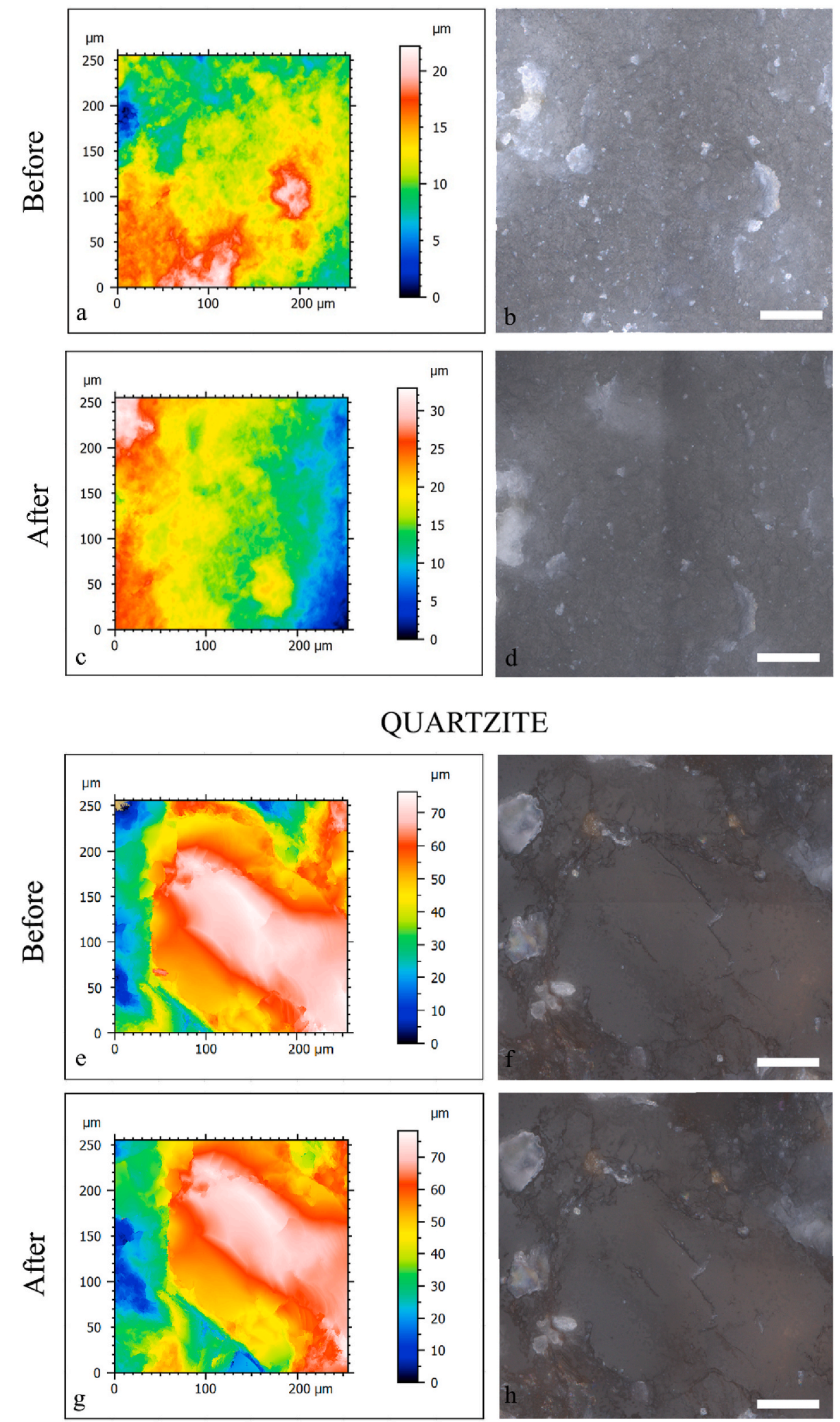

Fig. 2. Comparison of two areas of a flint and a quartzite tool acquired before and after performing the experiment. Top: FLT3-10-Area 1 (brushing + sediment); Bottom, QTZ3-3-Area 1 (gentle removal of sediment through rubbing). a, c, e, g) Topographic images where colors indicate height; b, d, f, h) Bright field microscopic images. Scale bars: $50 \mu \mathrm{m}$. (For interpretation of the references to color in this figure legend, the reader is referred to the Web version of this article.) 
which are included in a ConfoMap (v7.4.8964) template, a derivative of MountainsMap Imaging Topography (Digital Surf, Besançon, France) that was applied to all 3D surfaces $(n=30)$ (Fig. 3):

1. Loading the topography surface;

2. Leveling (Least squares method by subtraction);

3. Form removal (polynomial of degree 3);

4. Outliers removal (maximum slope of $80^{\circ}$ );

5. Thresholding the surface between 0.1 and $99.9 \%$ material ratio to remove the aberrant positive and negative spikes;

6. Applying a robust Gaussian low-pass $\mathrm{S}$-filter $\left(\mathrm{S}_{1}\right.$ nesting index $=$ $0.425 \mu \mathrm{m}$, corresponding to about 5 pixels, end effects managed) to remove noise;

7. Filling-in the non-measured points (NMP), necessary for the computation of some parameters;

8. Calculating SSFA parameters epLsar, Asfc, Smfc, HAsfc9 and HAsfc81 (according to Scott et al., 2006);

9. Applying a robust Gaussian high-pass L-filter (L nesting index $=$ $127 \mu \mathrm{m}$, corresponding to about half the FOV in X or Y direction, end effects managed) to separate the waviness (S-F surface) from the roughness (S-L surface);
10. Calculation of 21 ISO 25178-2 parameters (International Organisation for Standardisation, 2012), 3 furrow parameters, 4 texture direction parameters, and 4 texture isotropy parameters from both the roughness (S-L) and waviness (S-F) surfaces.

11. Altogether, 69 parameters were calculated on each surface.

The ConfoMap templates for each surface in MNT and PDF formats (including all original and processed surfaces, as well as all results) are freely available on Zenodo (https://doi.org/10.5281/zenodo.3632490).

\subsection{Statistical analysis}

Preparation of the data and all descriptive analyses (summary statistics and scatter plots) were performed in the open-source software $\mathrm{R}$ (v. 3.6.2; R Core Team, 2019) through RStudio (v. 1.2.5019; RStudio Team, 2019) for Microsoft Windows 10. The following packages were used: chron (v. 2.3-54; James and Hornik, 2020), doBy (v. 4.6-3; Højsgaard and Halekoh, 2020), ggplot2 (v. 3.2.1; Wickham, 2016), openxlsx (v. 4.1.4; Schauberger and Walker, 2019), and R.utils (v. 2.9.2; Bengtsson, 2019). Scripts, results and reports of the analyses in HTML format, created with knitr (v. 1.26; Xie, 2014, 2015; Xie et al., 2018) and rmarkdown (Xie et al., 2018; v. 2.0; Allaire et al., 2019), are freely
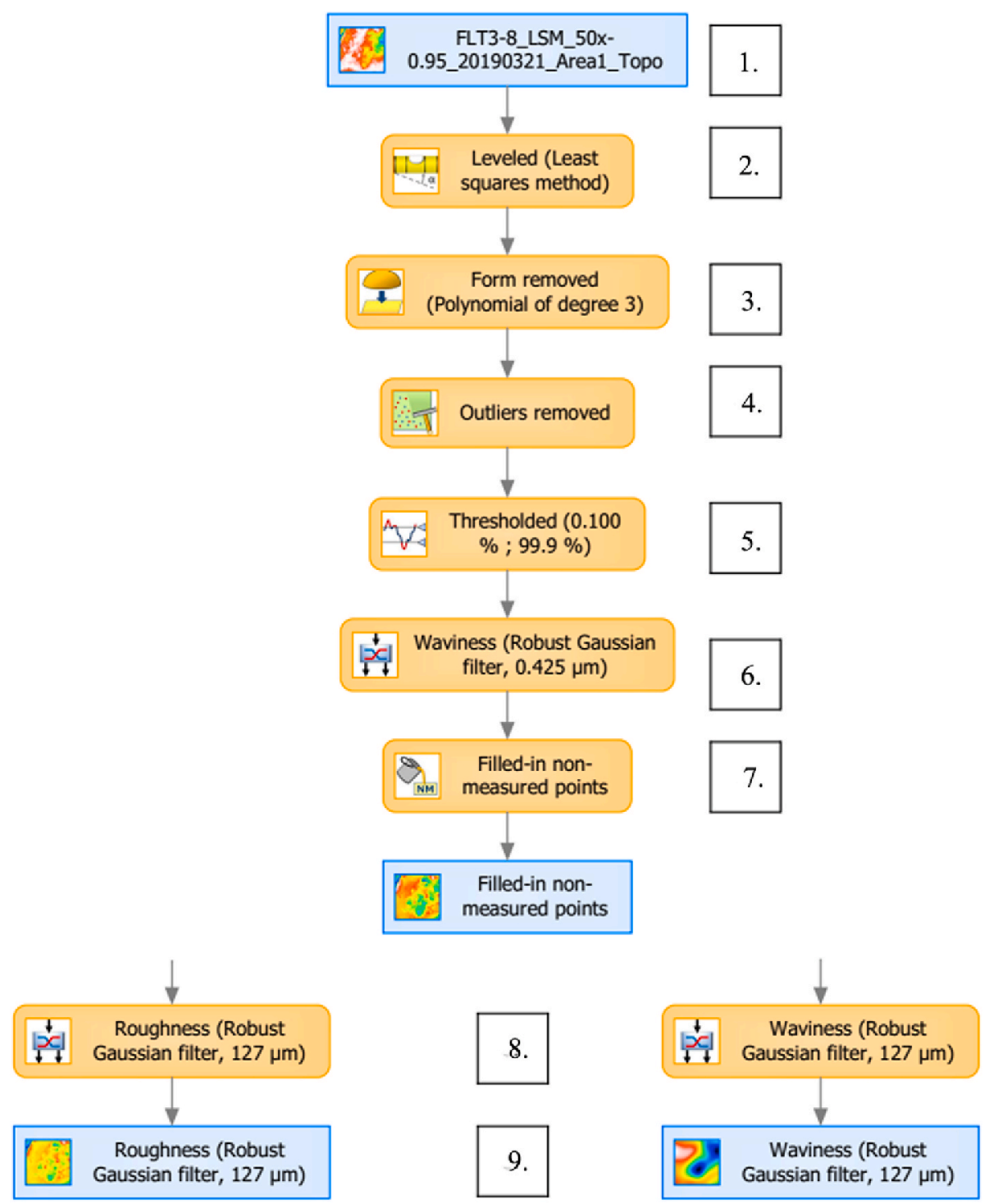

Fig. 3. Analysis workflow for the processing of surface data. 
available on Zenodo (https://doi.org/10.5281/zenodo.3632517).

All parameters applied to the waviness (S-F) surfaces were appended the suffix ".SF", while those applied to the roughness (S-L) surfaces were appended the suffix ".SL" (Step \#4.6 of the R script \#1).

Note that there are two Isotropy parameters, one from the texture direction analysis and one from the texture isotropy analysis, each calculated on both S-F and S-L surfaces. R requires 'data.frames' to have unique headers and " 1 " was appended automatically at the end of the second parameter, so that the name of the texture isotropy parameter was changed to 'Isotropy.SF.1' and 'Isotropy.SL.1' (Step \#4.7 of the R script \#1).

Six parameters (Periodicity, Period, and Direction of Period, on both $S$ - $F$ and $S$ - $L$ surfaces) could not be calculated on all surfaces so they were excluded from the subsequent Bayesian analysis, resulting in 63 parameters being analyzed.

To evaluate whether the different cleaning procedures change the measured value of the surface parameters significantly, a Bayesian Multi-factor ANOVA was applied. This method computes the amount of variance that can be attributed to a single factor, i.e. an independent variable, or a combination of two factors using Bayesian inference. There are several advantages to this approach compared to the traditional null hypothesis testing procedure (Kruschke, 2013). First, this method does not rely on assumptions other than the ones stated below and is, therefore, more transparent. Second, and more important, by using the full posterior distribution for the significance testing, the certainty of the results can also be assessed. Finally, regarding the practical component of the analysis, the availability of steadily increasing computational power and user-friendly software libraries means that the greater complexity of the computation should not present a serious drawback relative to the gain in insight.

Before performing the Bayesian Multi-factor ANOVA, the values of each parameter were transformed into standard scores, i.e. the sample mean was subtracted and the result was divided by the sample standard deviation. There are two reasons for this. First, from a theoretical point of view, this enables the use of a single model for all parameters, which in turn improves comparability. Second, from a computational point of view, having all values involved in a computation in a narrow numerical range stabilizes the algorithm against numerical errors. Note, however, that while the description of the model below is expressed in standard scores, the estimated model parameters were scaled back to the original numerical ranges for the plots, which allows better comparison.

The cleaning procedure, i.e. treatment (Control, BrushDirt, RubDirt, BrushNoDirt, see Table 1 ) is considered here as the first factor, $x_{1}$, while the type of raw material (quartzite or flint) is considered as the second factor, $x_{2}$. For every single measured surface parameter, the expected difference between the measurement outcomes after and before the procedure, $\mu$, is related to the factors by a linearized model:

$\mu=\beta_{0}+\beta_{1} \cdot x_{1}+\beta_{2} \cdot x_{2}+x_{1} \cdot M \cdot x_{2}$

The terms of the equation can be understood as follows: $\beta_{0}$ is a real number that indicates the overall order of magnitude of the measured values. $\beta_{1}$ is a vector of length 4 that contains the effect strengths of choosing the cleaning procedure, while $x_{1}$ is a vector that indicates the level of factor 1 , i.e. $x_{1}$ is $[0,0,0,1]$ when choosing the first level of factor $1=$ Control (no brushing and no dirt). The same applies to $\beta_{2}$ and $x_{2}$, but here with 2 different levels for quartzite and flint. $M$ is a matrix where the entry $M_{i, j}$ indicates the effect strength of the particular combination of the two factors.

The model hence enables the attribution of effect strength to each of the variable treatment and the raw material separately. In order to check for a significant effect, the unknown model parameters $\beta_{0}, \beta_{1}, \beta_{2}$ and $M$ must be inferred from the data and the prior knowledge on the measurement process.

The observed difference between the measurement outcomes after and before the procedure, $y$, is the input to the model and its relation to the model prediction is assumed to be:

$\mathrm{y} \sim \mathrm{N}(\mu, \varepsilon)$

where ' $\sim$ ' means 'is distributed as' and $N(a, b)$ denotes a normal distribution with mean $a$ and standard deviation $b$.

For every unknown model parameter, the Bayesian framework requires the specification of the prior knowledge on that parameter, which was chosen as follows:

$\beta_{0 i} \sim N\left(0, \sigma_{0}\right)$

$\beta_{1 i} \sim N\left(0, \sigma_{l}\right)$

$\beta_{2 i} \sim N\left(0, \sigma_{2}\right)$

$M_{i, j} \sim N\left(0, \sigma_{M}\right)$

$\varepsilon \sim U\left(0\right.$, Error $\left._{\text {Max }}\right)$,

where $U(a, b)$ denotes a uniform distribution between $a$ and $b$. Indices refer to the fact that every component of a vector or matrix valued quantity is modeled as an independent distribution.

To make the prior distributions less informative, the parameters of the prior distributions are modeled as random variables themselves:

$\sigma_{0} \sim H N\left(s_{0}\right)$

$\sigma_{l} \sim H N\left(\mathrm{~s}_{1}\right)$

$\sigma_{2} \sim H N\left(s_{2}\right)$

$\sigma_{M} \sim H N\left(s_{M}\right)$,

Error $_{\text {Max }} \sim H N\left(s_{E}\right)$,

where $H N(s)$ denotes a half-normal distribution with standard deviation $s$

The parameters describing the distribution of the prior distribution parameters are called hyperparameters and are chosen as follows. $s_{0}$ is chosen as $1 . s_{1}$ and $s_{2}$ are calculated as the maximum observed effect strength when varying factor 1 or 2 , respectively. $s_{M}$ is computed as $5 \%$ of the combined effect strength $\sqrt{s_{1}^{2}+s_{2}^{2}}$ as, from a priori knowledge, there is no strong multiplicative interaction between the cleaning procedure (i.e. treatment) and the raw material type. Assuming a strong multiplicative interaction would imply that a general (i.e. independent of raw material) statement about the effect of a cleaning procedure cannot be made, but the effect of a cleaning procedure is only meaningful in the context of a specific raw material. $s_{E}$ is chosen as $10 \%$ of the minimum of $s_{1}$ and $s_{2}$, although the measurement process itself is far more precise.

The posterior distribution is now accessed by sampling using a special variant of Markov Chain Monte Carlo, the Hamiltonian Monte Carlo algorithm (Hoffman and Gelman, 2014) in the implementation by Salvatier et al. (2016). When performing the sampling, the results have to be checked for consistency based on the trace plots and on the energy plots of Hamiltonian Monte Carlo (see Supplementary Material for details, http://doi.org/10.5281/zenodo.3873124). After having computed the samples from the posterior, the so-called contrast (i.e. the distribution of the differences between the components describing a cleaning procedure and the component of $\beta_{1}$ describing the control group) can be analyzed. To decide whether there is a significant effect between the cleaning procedures, the $95 \%$ high probability density interval (HPD) of $2.5 \%-97.5 \%$ cumulated probability of the contrast is considered. If zero effect strength is not within that interval, the effect is considered significant. Remember that the model describes the influence of both treatment and raw material, but divides them into two independent contributions. Therefore, by comparing the components of $\beta_{1}$ instead of the raw values grouped by treatment, the influence of the raw material is implicitly taken into account but computationally removed in the 
contrasts. We did not consider the influence of the raw material alone for two reasons. First, the hypothesis we test concerns the treatments, not the difference between flint and quartzite. And second, due to the mineralogical, structural and topographical differences between these two raw materials, the model would surely find differences in textures. But again, these differences would not help address the questions of this study.

To check whether the modeling assumptions and estimated model parameter values are sensible, the prior and posterior predictive distributions were inspected. They describe the expected distribution of input data based on the prior and posterior distributions of the model parameters respectively. They were plotted on top of the input data distribution (http://doi.org/10.5281/zenodo.3873124). As the prior predictive distributions are considerably broader than the range of the input data, they can indeed be considered weakly informative. The posterior predictive distributions are well aligned with the input data and span a similar range; thus, the model parameters estimates are also considered sensible.

The whole analysis was performed in Python (3.7.3) with the package PyMC3 (Salvatier et al., 2016). Scripts and results are freely available on Zenodo (http://doi.org/10.5281/zenodo.3873124).

\section{Results}

The samples are divided into four different categories according to the treatment they have been subjected to. They are defined by two terms, the first referring to the brushing activity, the second to the application of dirt (i.e., standard sediment). Hence, we have: 1) "Control": No brushing and No dirt, i.e. control samples (blue color on the plots); 2) "BrushNoDirt": Brushed but No dirt (orange); 3) "RubDirt": No brushing but Dirt applied, i.e. rubbing (green); 4) "BrushDirt": Brushed and Dirt applied, i.e. the standard sediment was brushed off (red). This notation and the color labels are used throughout the text, figures and tables. In the Python analysis, the nomenclature differs as the analysis was run using different labels. Hence, in the supplementary material (http://doi.org/10.5281/zenodo.3873124) we have: 1) No_ No = Control; 2) Is_No = BrushNoDirt; 3) No_Is = RubDirt; 4) Is_Is = BrushDirt.

Since our goal is to test whether these mechanical actions alter the surface texture, we focus here on the parameters that changed significantly after treatment, as compared to the controls. The significant differences are shown in Table 2 and a description of the parameters for which a significant difference was found is given in Table 3. Note that the parameter Isotropy from the Texture Direction analysis (Isotropy.SF/ Isotropy.SL) is identical to the ISO 25178 Str parameter (Isotropy $=100$ $\times$ Str according to the ConfoMap help guide), and is therefore not shown nor counted in the number of significant differences; it appears in the overview (Table 2) though.

Figs. 4-11 show the plots only for the parameters and contrasts that changed significantly. Nevertheless, all plots for all parameters are available as supplementary materials on Zenodo (Script3_plots.html on https://doi.org/10.5281/zenodo.3632517, and PDF files on http://doi. org/10.5281/zenodo.3873124).
The two left scatterplots on Figs. 4-11 show the measured values separating flint from quartzite to highlight possible divergences.

For the analysis, the samples pertaining to the categories other than the controls were tested against the controls themselves, which were assumed to be constant. In this phase, the delta values $(\Delta)$, i.e. the differences between the values of the two acquired surfaces (value ${ }_{\text {after }}$ value $_{\text {before}}$ ), are considered. For the control samples, $\Delta$ values are expected to be around 0 , and this is what was generally observed.

Note that the data points are not grouped according to raw material type here, but are solely grouped by the treatment applied which could of course mask differences dependent on the raw material. However, this problem is solved by the application of the model described in the method section, so that the effects that are dependent on the treatment (but independent from the raw material) can be studied.

For those treatments with a significant effect, the right plot in Figs. 4-11 shows the estimated distribution of differences in $\Delta$ values due to the treatment. In these so-called "Contrast plots" the $95 \%$ high probability density interval (HPD) (black horizontal bar with extreme values; see also Table 2) is shown. If this interval does not include 0 (orange vertical bar), the treatment (BrushNoDirt, RubDirt or BrushDirt) is considered to have a significant effect relative to the control (Control).

The most significant differences (7 out of 10) occurred when only brushing (BrushNoDirt) was performed, as compared to the control. Only one parameter changed significantly when the standard sediment was removed via mechanical brushing (BrushDirt), as compared to the control. The last two differences are between rubbing (RubDirt) and the control samples.

Anisotropy (epLsar) is the only parameter that detects significant differences between brushing the sediment off (BrushDirt) and the control samples (Control). Additionally, differences between brushing without dirt (BrushNoDirt) and control samples were found for this parameter (Fig. 4).

Three different isotropy parameters changed significantly - Isotropy. SF, Isotropy.SF. 1 and Str.SF - whereby the first and the last are identical (see above). All changed significantly only when brushing without dirt (BrushNoDirt) (Figs. 5-6), Anisotropy (as measured by epLsar) is the reverse of isotropy (as measured by Isotropy.SF, Isotropy.SF.1 and Str.SF). When combining all these (an)isotropy parameters, it seems brushing without dirt decreases the directionality of the surface textures (i.e. isotropy increases and anisotropy decreases).

In general, quartzite seems to behave differently from flint. It is particularly visible when rubbing the surfaces (Fig. 5), and even when brushing with dirt is considered (Fig. 6). This might explain why there are no stronger signals in the analysis (where both raw materials are combined).

Values of Vvv.SF (dale void volume) change significantly between RubDirt and Control samples, as well as between BrushNoDirt and Control (Fig. 7), with both treatments lowering the values. It seems that the quartzite samples have generally higher values than flint.

Smr.SL (areal material ratio of the scale-limited surface) is significantly different for the samples that have been rubbed (RubDirt),

Table 2

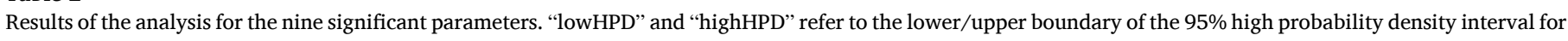
each treatment (RubDirt, BrushNoDirt and BrushDirt) relative to the Controls.

\begin{tabular}{|c|c|c|c|c|c|c|c|c|c|}
\hline Variable & RubDirt & lowHPD & highHPD & BrushNoDirt & lowHPD & highHPD & BrushDirt & lowHPD & highHPD \\
\hline epLsar & False & -0.00048 & 0.00002 & True & -0.00068 & -0.00011 & True & -0.00069 & -0.00013 \\
\hline$H A s f c 9$ & False & -0.03597 & 0.46531 & $\overline{\text { True }}$ & 0.05645 & 0.66560 & $\overline{\text { False }}$ & -0.02149 & 0.48796 \\
\hline Sku.SL & False & -0.95614 & 0.71538 & $\overline{\text { True }}$ & 0.29304 & 2.01230 & False & -1.58801 & 0.04914 \\
\hline Smr.SL & True & 0.00974 & 0.33128 & $\overline{\text { False }}$ & -0.15906 & 0.12710 & False & -0.19593 & 0.09021 \\
\hline Sxp.SF & $\overline{\text { False }}$ & -1140.30951 & 59.36720 & True & -1480.34277 & -79.20018 & False & -846.68490 & 332.44626 \\
\hline Str.SF & False & -0.32471 & 0.02336 & $\overline{\text { True }}$ & 0.23673 & 0.60343 & False & -0.10513 & 0.24846 \\
\hline$V v v \cdot S F$ & True & -0.09911 & -0.01902 & $\overline{\text { True }}$ & -0.12113 & -0.03600 & False & -0.07054 & 0.00531 \\
\hline Isotropy.SF & $\overline{\text { False }}$ & -33.36246 & 2.72304 & $\overline{\text { True }}$ & 22.19371 & 58.36145 & False & -11.78607 & 24.52500 \\
\hline Isotropy.SF.1 & False & -23.94113 & 13.65518 & $\overline{\text { True }}$ & 4.08876 & 46.50621 & False & -1.82528 & 37.04306 \\
\hline
\end{tabular}




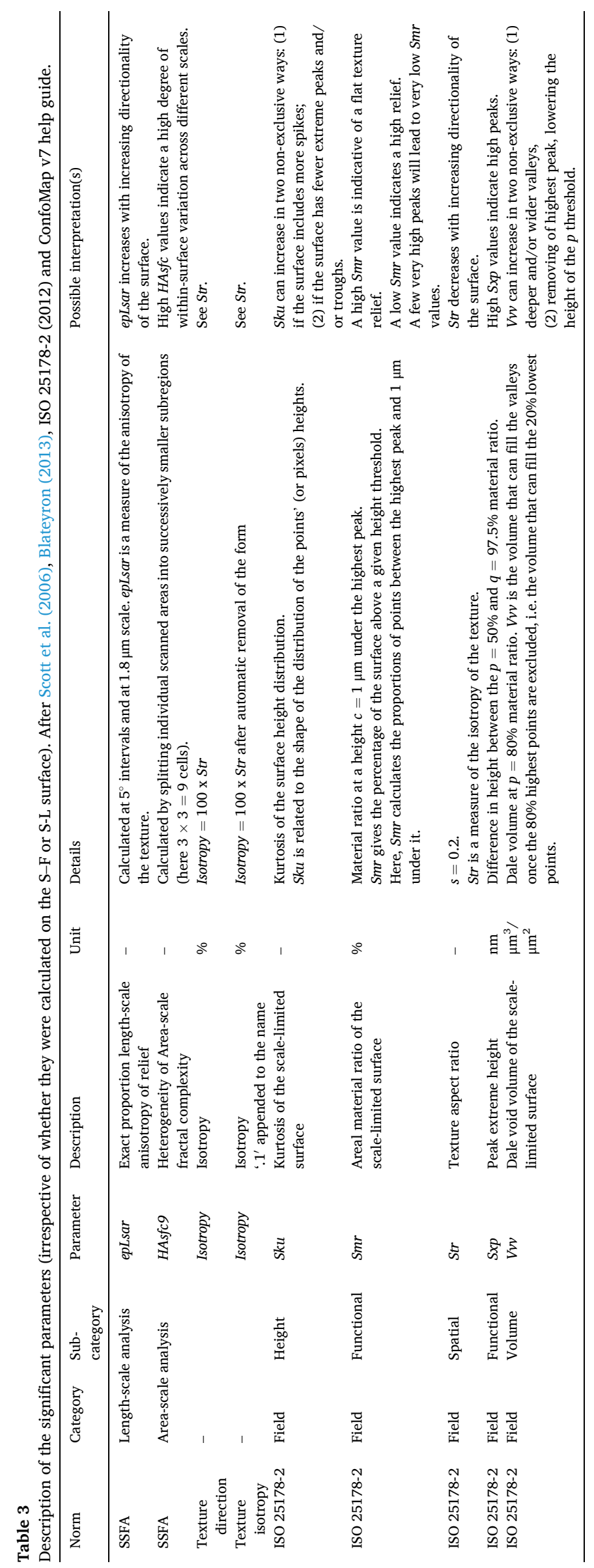

compared to the controls (Control). Changes are more visible in the flint samples, while the quartzite ones seem to be more stable (Fig. 8).

All other significant parameters (HAsfc9, Sxp.SF and $S k u . S L$ ) changed only between brushing without dirt (BrushNoDirt) and control samples (Figs. 9-11).

\section{Discussion}

Although in the domain of traceology confocal microscopy has mainly been used to quantify use-related polish (e.g. Stemp et al., 2013; Ibáñez et al., 2014, 2018; Macdonald et al., 2018), it can also be used to quantify changes in surface textures due to other causes (e.g. post-depositional alterations; Vietti, 2016; Caux et al., 2018; Werner, 2018; Galland et al., 2019). There are multiple causes for the formation of wear. During manufacture, technological marks (e.g. knapping marks) (Kamminga, 1979; Cotterell and Kamminga, 1987), as well as hafting and de-hafting marks (Rots, 2010), can alter the surface of stone tools. Ancient use (e.g. Keeley, 1980) as well as past activities unrelated to use (such as carrying stone tools in the same bag in close contact one with another) can also modify the micro-topography of tools. Post-depositional surface modifications (PDSMs) (Levi Sala, 1986; McPherron et al., 2014) might compromise the functional reading of artifacts, as they cover possibly present use-wear. Archeologists can also leave unwanted traces on the surfaces of stone tools during both excavation and post-excavation treatment (such as cleaning procedures) (Pedergnana et al., 2016). Among laboratory analyses, the riskiest one to be carried out before use-wear analysis is certainly refitting analysis. There is a high chance of producing modern scratches on the surfaces of stone tools by rubbing pieces against each other while refitting.

Among this variety of causes for wear formation, we selected one to be tested: the removal of sediment particles from archeological stone tools. Brushing stone tool surfaces with brushes featuring plastic bristles and rubbing them with bare fingers are common cleaning procedures applied both on-site and in laboratories. They are performed to remove firmly adhering sediment particles that are attached to the surfaces of tools and that have to be removed prior to further analyses. It is particularly important to remove such particles when microscopic analyses of stone tool surfaces are envisaged.

In this contribution, we presented the results of an experiment designed to test whether mechanically removing sediment particles from stone tools modifies their micro-topography or not. A sediment mixture containing slaked lime, water and quartz grains was applied to some experimental samples. We are aware that it is not possible to cover all archeological scenarios by using this mixture. Ideally, different sediment mixtures should be tested, depending on the archeological assemblages under study. We decided to initially test one specific mixture, as the effect that brushing has on stone tools' surfaces was largely unknown.

Nine parameters ( $14 \%$ of the tested parameters; see "Notebook_Overview.html" on http://doi.org/10.5281/zenodo.3873124) changed significantly due to the mechanical actions applied to the samples as compared to the changes observed on the control samples. Most changes were observed when the surfaces were brushed without sediment (BrushNoDirt). The slight changes observed on the control samples (before vs. after) are interpreted to be the results of measurements errors and also on the $14 \%$ error of the coordinate system used to re-locate the areas on the samples (Calandra et al., 2019a).

General trends came up from the significant values measuring isotropy in the broad sense (Isotropy.SF, Isotropy.SF.1, Str.SF and epLsar) (Figs. 4-6). There is a general increase in isotropy when brushing without sediment at the scale of waviness (SF surface) and across scales (epLsar). This data could be explained as a general employment of variable directions of movement when using a toothbrush.

Unexpectedly, the combination of sediment and brushing (BrushDirt) was found to be significant only for anisotropy (epLsar), as compared to the control samples (Control). This could be due to at least 

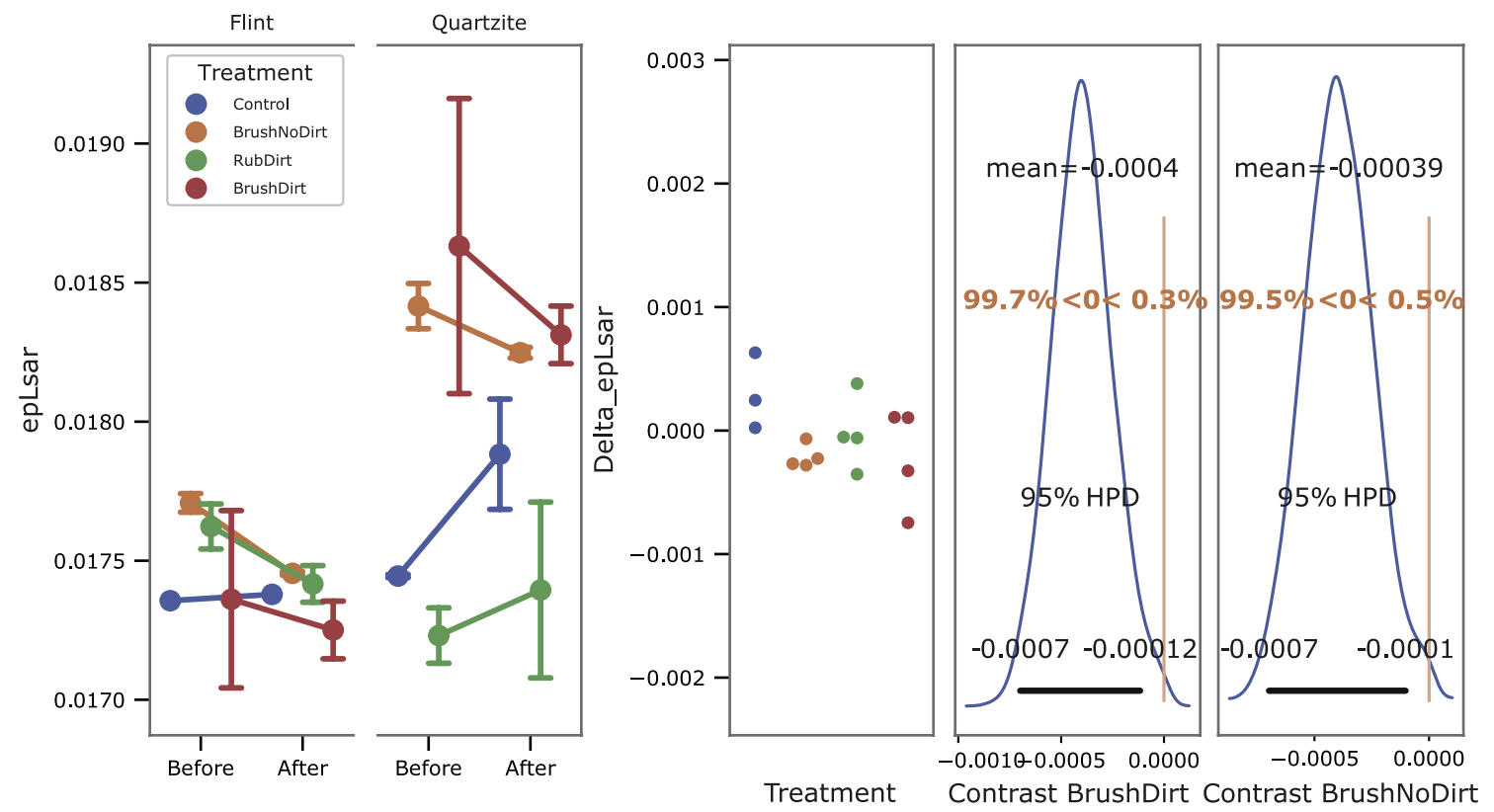

Fig. 4. From left to right: Plot of the original values for epLsar for each of the two raw materials. The middle graph shows the differences of the values acquired before and after the experiment (delta $=$ value $_{\text {after }}-$ value $_{\text {before}}$ ) and the last graph is a contrast plot showing the significance for this parameter (epLsar is significant when samples were brushed, i.e. BrushDirt and BrushNoDirt).
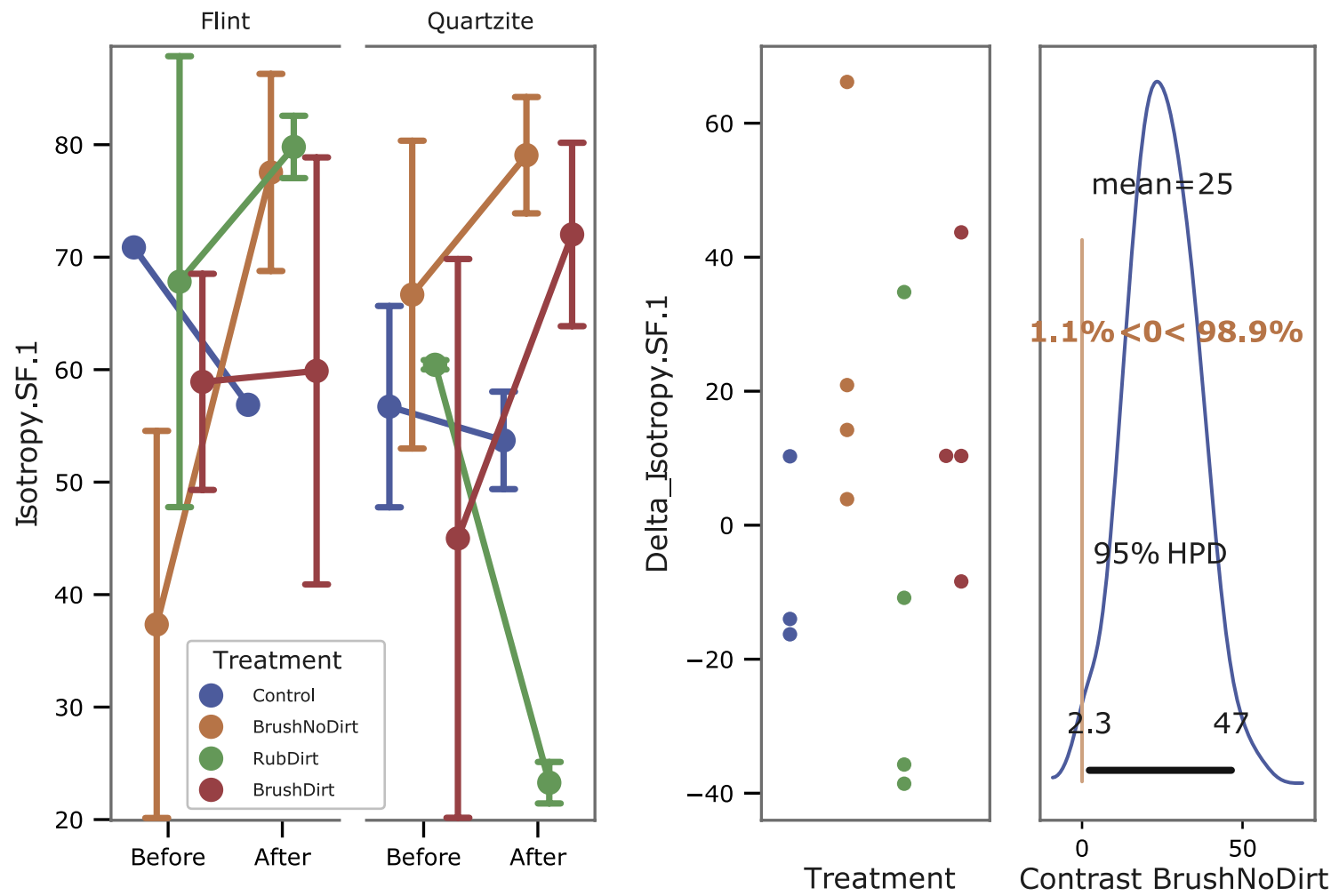

Fig. 5. Plots of Isotropy.SF.1. This parameter is significant for the BrushNoDirt treatment.

three complementary effects. First, the larger the variation, the less likely it is that any statistical analysis will detect differences, especially with small sample sizes; it could for example explain why the BrushDirt treatment is not significantly different from the control for Str.SF (Fig. 6). Second, the effects of brushing alone (BrushNoDirt) and rubbing alone (RubDirt) are sometimes opposite, explaining why the combination might not be significant (e.g. Str.SF; Fig. 6). Lastly, flint and quartzite seem to behave differently for some parameters (e.g. epLsar and Isotropy.SF.1 for RubDirt, and Smr.SL for BrushNoDirt). This could be responsible for the generally weak signals measured and, therefore, could partially hide some potential effects from the different treatments. The higher values Vvv.SF (Fig. 7), Hasfc9 (Fig. 9) and Sxp.SF (Fig. 10) on the quartzite samples are likely to be the result of the general topography (large grains) of quartzite as opposed to the smooth surfaces of flint, at least at the waviness scale. The increase in heterogeneity $(H A s f c 9)$ is likely due mainly to quartzite, where wear processes are less 


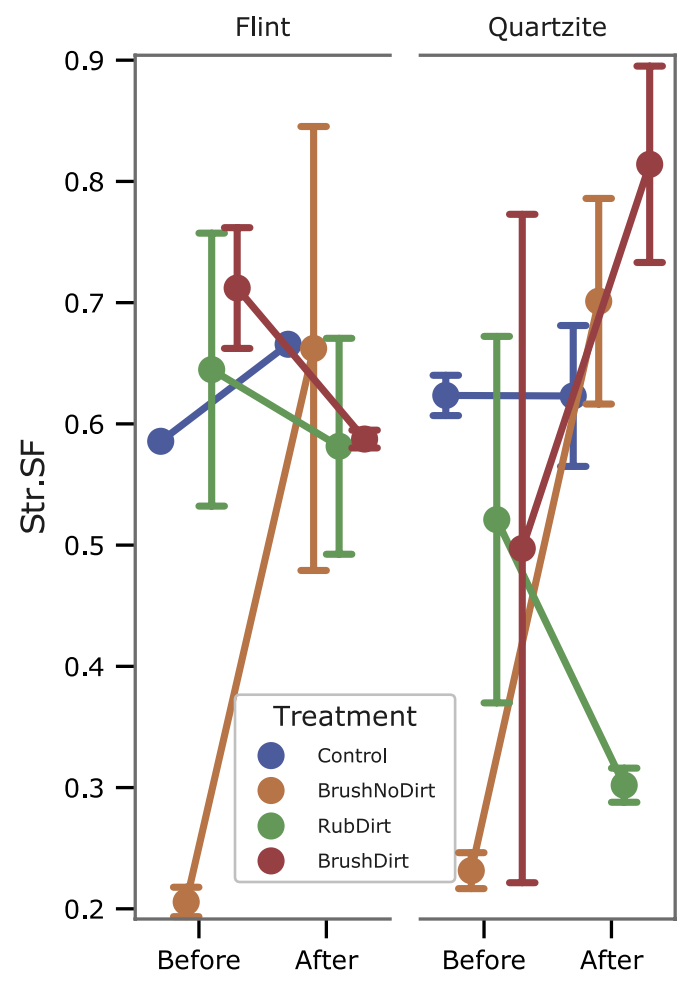

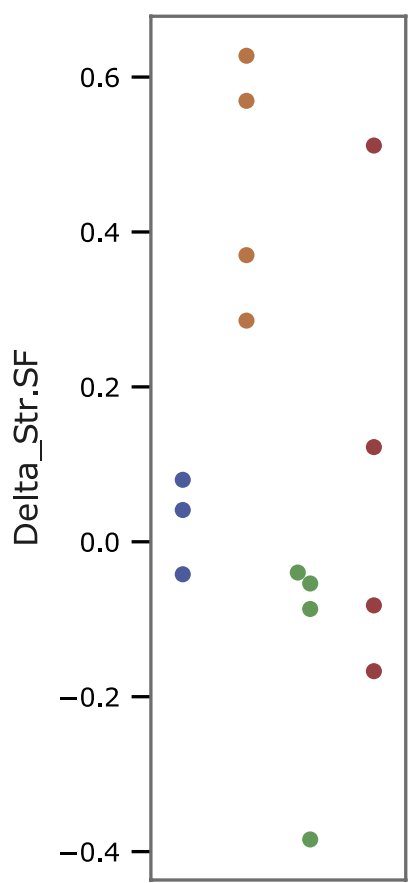

Treatment

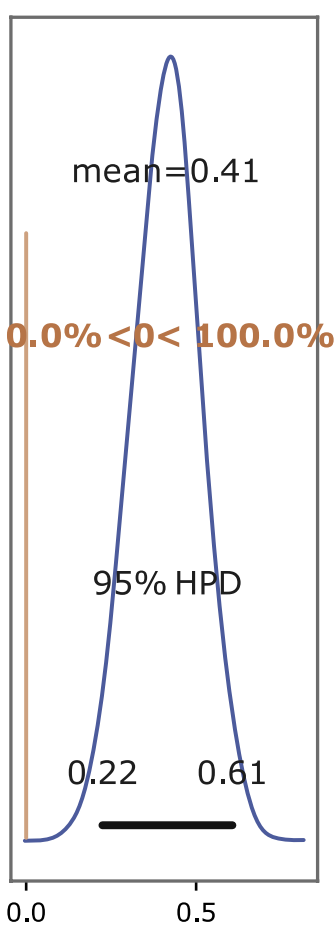

Contrast BrushNoDirt

Fig. 6. Plots of Str.SF = texture aspect ratio. This parameter is significant for the BrushNoDirt (only brushing) treatment.

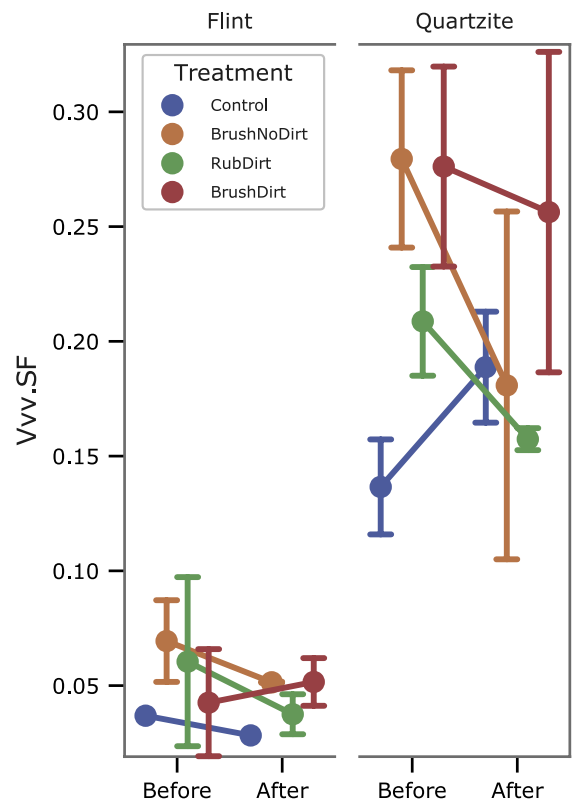

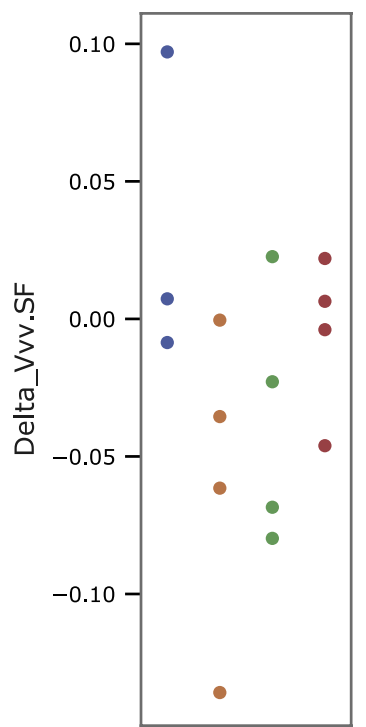

Treatment
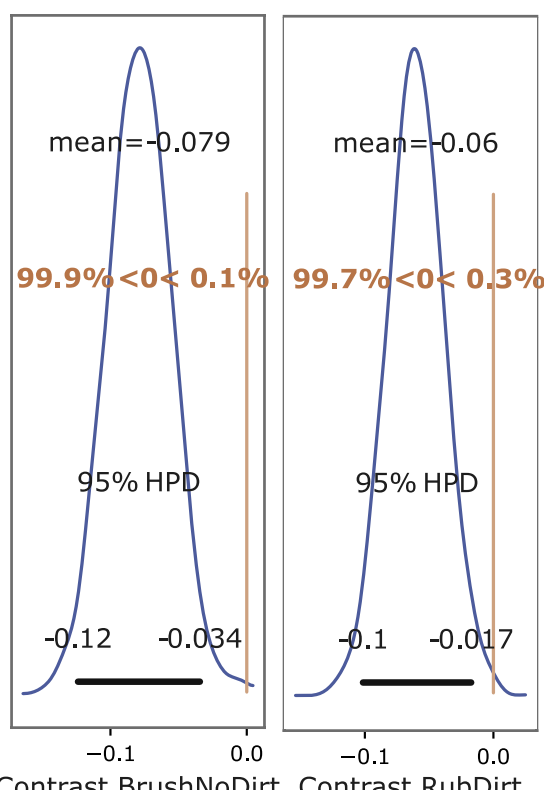

Fig. 7. Plots of $V v v \cdot S F=$ dale void volume. This parameter is significant for two treatments: BrushNoDirt (only brushing) and RubDirt(rubbing).

evenly distributed over the surface than in flint (Clemente-Conte and Gibaja-Bao, 2009; Pedergnana and Ollé, 2017). The decrease in Vvv.SF and Sxp.SF after brushing without dirt (significant for both parameters) and rubbing (significant only for $V v v . S F$ ) in both raw materials could be explained by abrasion. $V v v$ is calculated after exclusion of the $80 \%$ highest points (Table 3). When the highest peaks are removed through abrasion (decrease in $S x p . S F$ ), the exclusion threshold moves down toward the lowest points, leading to the calculation of the volume of shallower dales (pits) with smaller volumes (lower Vvv.SF values).
$S m r . S L$, together with $V v v . S F$, are the only parameters that detected significant changes between rubbing (RubDirt) and controls (Fig. 8). Rubbing tends to decrease the texture relief at the roughness scale (higher Smr.SL values after the experiment, SL surface), which could be due to abrasion, a phenomenon already hinted by the $V v v . S F$ patterns (see above). Flint appears to have less roughness relief (higher Smr.SL values) in general than quartzite. Rubbing tend to reduce this relief even further on flint, while the quartzite samples change to a lesser extent. It could be that the few peaks present on the flint abrade more easily, 

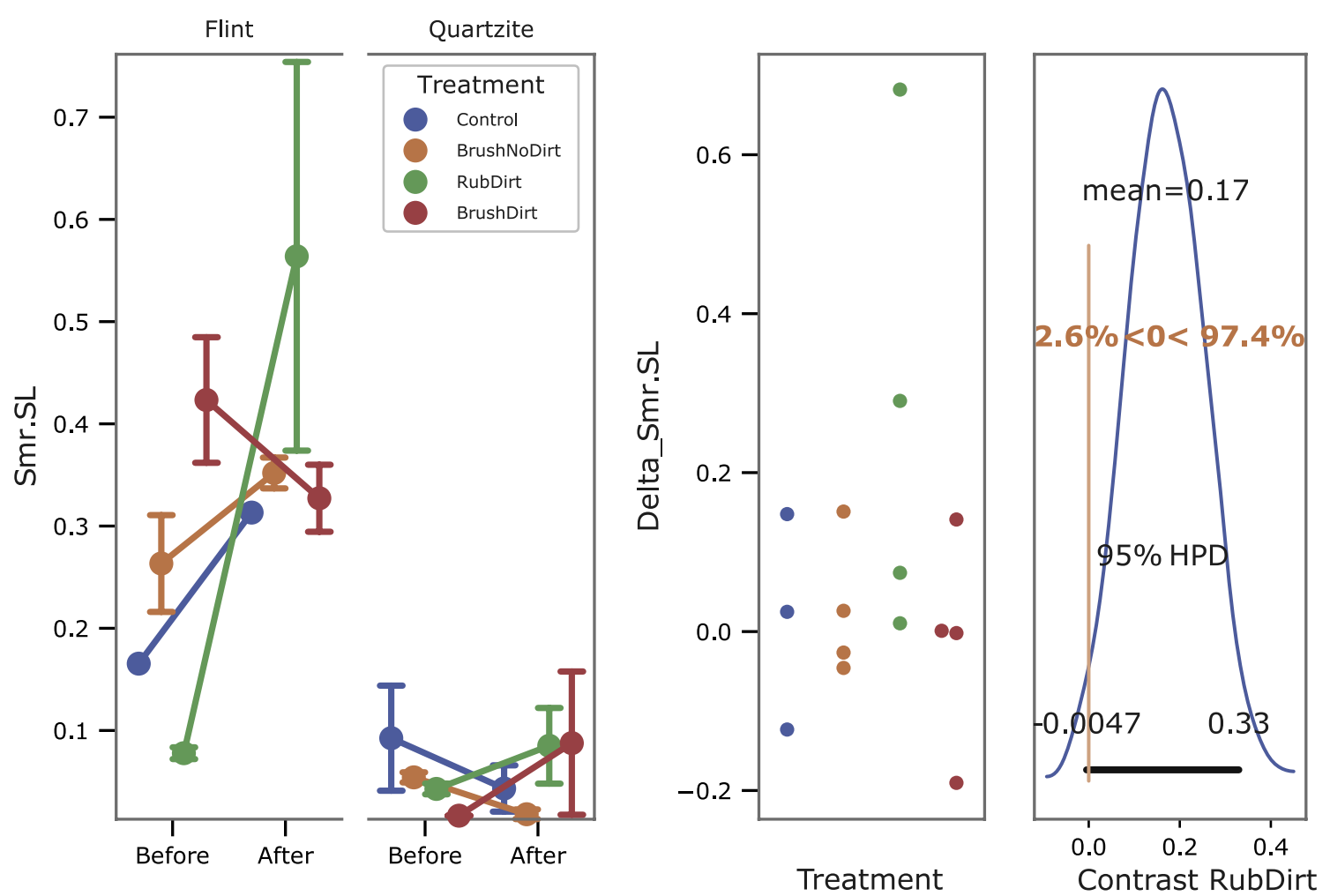

Fig. 8. Plots of Smr.SL = areal material ratio of the scale-limited surface. This parameter is significant for the RubDirt treatment (rubbing).

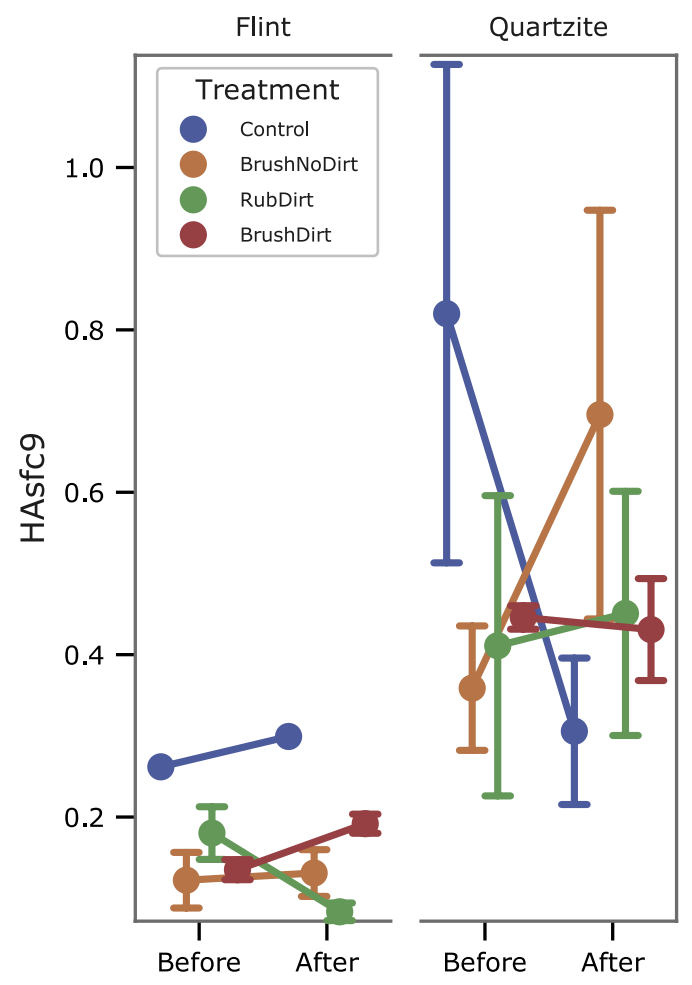

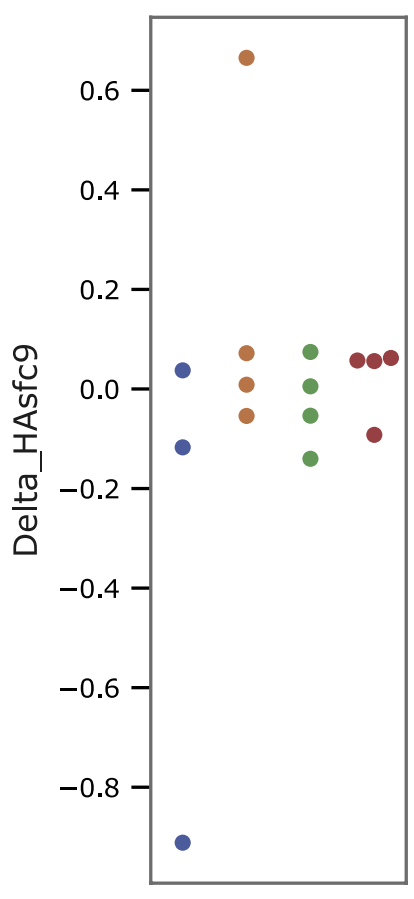

Treatment

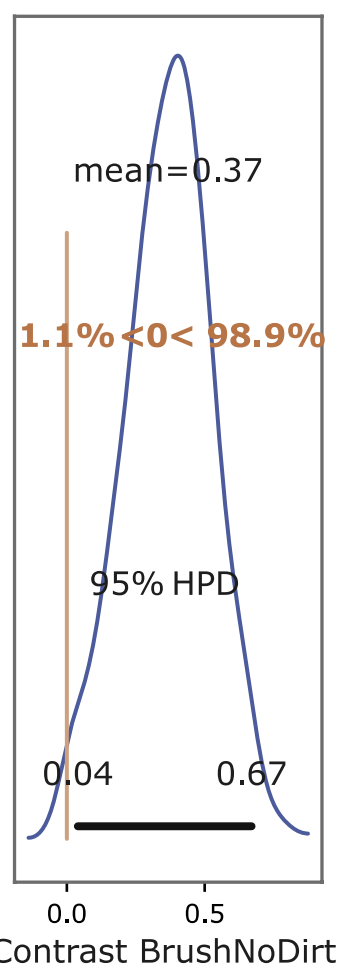

Fig. 9. Plots of $H A s f c 9=$ Heterogeneity of Area-scale fractal complexity: This parameter is significant for the BrushNoDirt (only brushing) treatment.

leading to large changes. On the other hand, abrading or dislodging quartz grains from the quartzite does not change the general roughness relief significantly.

The kurtosis of the surface height distribution (Sku.SL) detected significant changes when brushing without dirt at the roughness scale (Fig. 11). Both materials seem to become spikier and/or have fewer extreme peaks/troughs, although the change is larger for flint.

Even though these results are preliminary, the take-home message is 

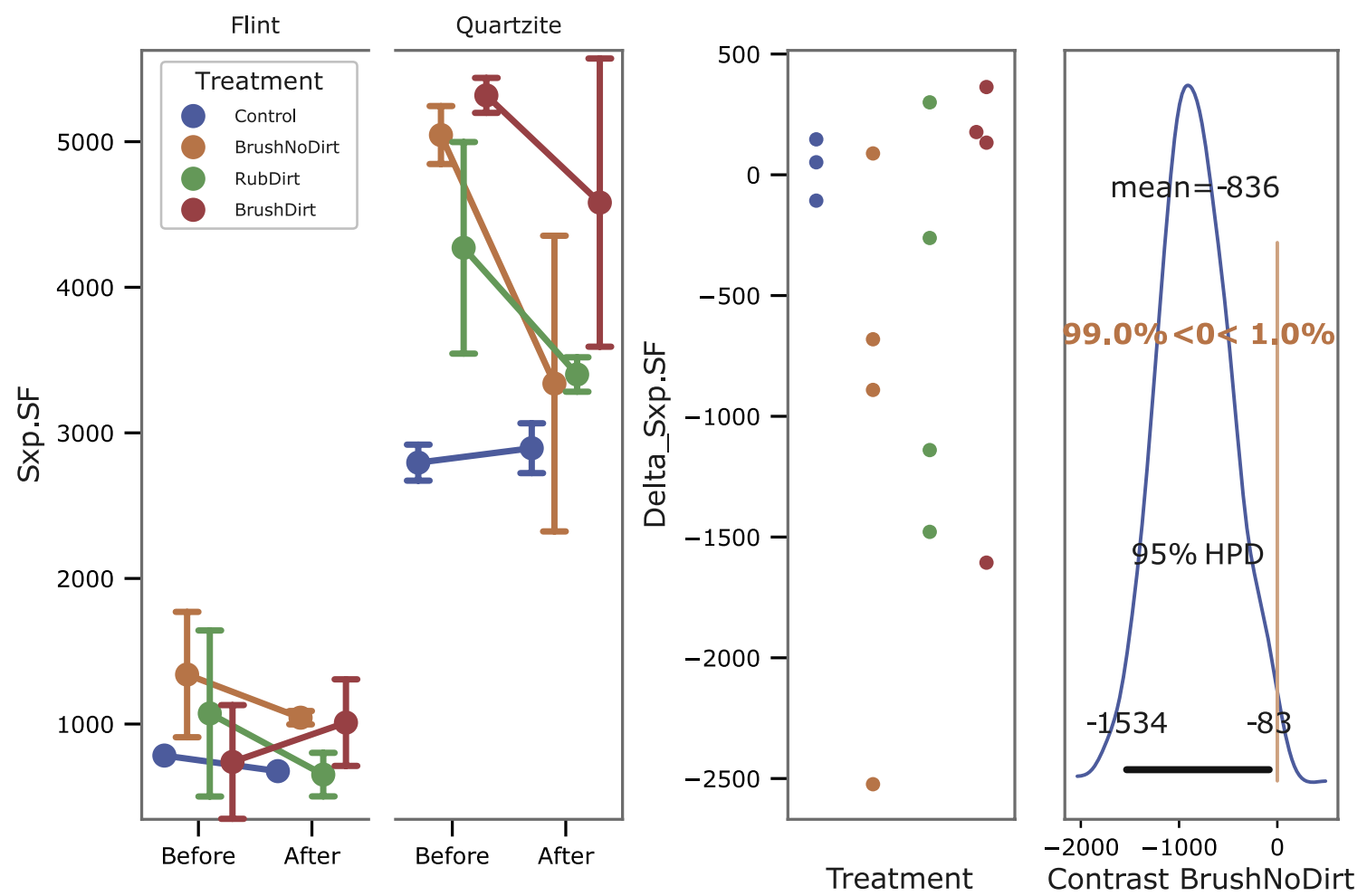

Fig. 10. Plots of Sxp.SF = Peak extreme height. This parameter is significant for the BrushNoDirt (only brushing) treatment.
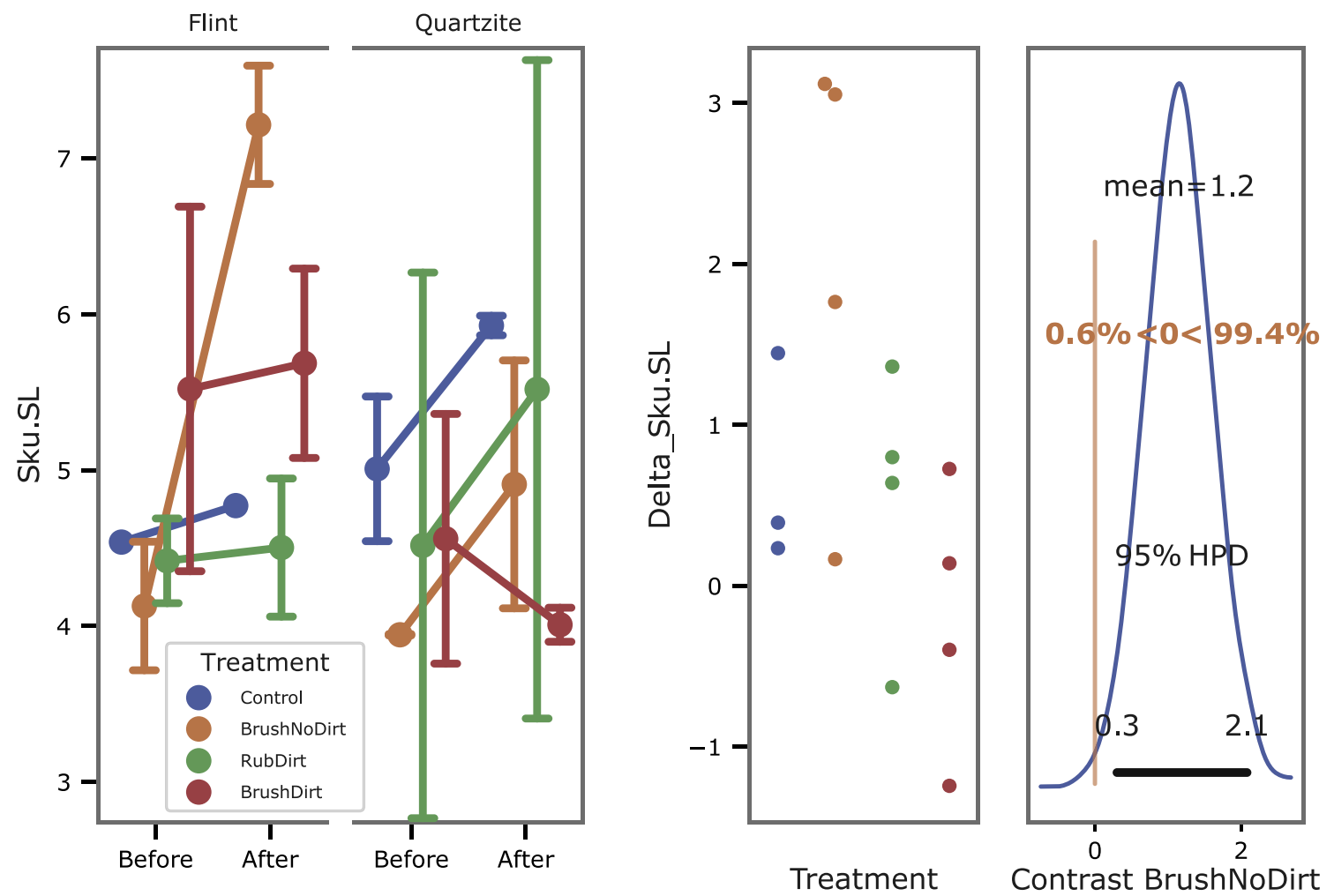

Fig. 11. Plots of $S k u . S L=$ kurtosis on the roughness surfaces. This parameter is significant for the BrushNoDirt treatment (only brushing).

that such light mechanical actions do change the surfaces in measurable ways. Brushing by itself seems to have the largest impact, but the removal of sediment by rubbing or brushing also modified the surfaces to a lesser extent. We therefore recommend cautious cleaning, avoiding hard and long actions as far as possible. Nevertheless, it is currently unknown how these modifications compare to surface modifications due to use (i.e., use-wear) on different worked materials; it is likely that userelated polish has a much stronger signature and that the surface modifications due to cleaning would simply disappear in the noise. It also remains to be tested whether all parameters are affected the same way 
by cleaning and by use.

To better understand the effects of the mechanical actions described here on different raw materials, more work is certainly needed. The fact that the analysis did not provide general strong signals is interpreted partly as a consequence of the small sample size (only one object per treatment and per raw material). A possible solution for highlighting differences in the two raw materials could be to perform sequential experiments seeking to understand when most of the textural changes happen as well as enlarging the sample size.

Also, different sediments (different granulometry and composition) and longer brushing/rubbing times should be tested, together with improved directional control. The fact that more parameters are significant for the brushing action than for rubbing might indicate a general higher pressure applied with the tooth brush, with more irregular motions. The movements were not continuous and regular, as they probably constantly changed directions (and pressure) while focusing on the areas where dirt persisted (therefore, avoiding those already free from dirt).

As brushing is just the first, mechanical step of most cleaning protocols adopted both on-site and in laboratories, other cleaning procedures should be tested. For example, the use of acid and basic solutions (at different concentrations) should be systematically tested in order to make sure that no additional changes are added to the surfaces of archeological artifacts (Macdonald and Evans, 2014). Even flint could be affected by acid solutions, because it can contain varying amounts of calcium carbonate inclusions (Hughes et al., 2012).

Furthermore, the effect of PDSMs has to be further studied (Caux et al., 2018; Werner, 2018; Galland et al., 2019), and more experiments involving the burial of stone tools in different sediment types are the way to go. It is indispensable to be able to correctly identifying PDSMs and eliminate them from functional interpretations. Unfortunately, they can overlap in morphology with traces due to use; more data on how to successfully identify them is therefore needed.

Moreover, large datasets of quantified surfaces (natural and worn down surfaces) are needed to define the most appropriate parameters to analyze surfaces made of different raw materials. It is possible that specific parameters have to be selected for, or excluded from, the analysis on given raw materials. This is also suggested by the results presented here. Additionally, analytical workflows have to be tailored and specific cut-offs must be thoroughly tested in order to refine the method.

\section{Data availability}

All data generated and/or analyzed during the current study are included in this published article and its Supplementary Material files, or are available on Zenodo (https://doi.org/10.5281/zenodo.3632490 for the ConfoMap analysis, https://doi.org/10.5281/zenodo.3632517 for the R analysis, and http://doi.org/10.5281/zenodo.3873124 for the Python analysis).

\section{Declaration of competing interest}

The Authors have no conflict of interest.

\section{Acknowledgements}

Research has been supported within the Römisch-Germanisches Zentralmuseum - Leibniz Research Institute for Archeology by German Federal and Rhineland Palatinate funding (Sondertatbestand "Spurenlabor") and is publication no. 6 of the TraCEr laboratory. The funding source had no involvement in study design; in the collection, analysis and interpretation of data; in the writing of the report; nor in the decision to submit the article for publication.

The authors want to thank Geoff Carver for language assistance and the two anonymous reviewers for their very helpful comments.

\section{Appendix A. Supplementary data}

Supplementary data to this article can be found online at https://doi. org/10.1016/j.quaint.2020.06.031.

\section{References}

Allaire, J.J., Xie, Y., McPherson, J., Luraschi, J., Ushey, K., Atkins, A., Wickham, H., Cheng, J., Chang, W., Iannone, R., 2019. Rmarkdown: Dynamic Documents for R. R package version 2.1 [WWW Document]. https://rmarkdown.rstudio.com. (Accessed 21 January 2020).

Arman, S.D., Ungar, P.S., Brown, C.A., DeSantis, L.R.G., Schmidt, C., Prideaux, G.J., 2016. Minimizing inter-microscope variability in dental microwear texture analysis. Surf. Topogr. Metrol. Prop. 4 https://doi.org/10.1088/2051-672X/4/2/024007.

Bengtsson, H., 2019. R.utils: Various Programming Utilities. R package version 2.9.2 [WWW Document]. https://CRAN.R-project.org/package=R.utils. (Accessed 21 January 2020).

Blateyron, F., 2013. The areal feature parameters. In: Leach, R. (Ed.), Characterisation of Areal Surface Texture. Springer Berlin Heidelberg, Berlin, Heidelberg, pp. 45-65. https://doi.org/10.1007/978-3-642-36458-7_3.

Brink, J.W., 1978. The role of abrasives in the formation of lithic use-wear. J. Archaeol, Sci. 5, 363-371.

Calandra, I., Schunk, L., Rodriguez, A., Gneisinger, W., Pedergnana, A., Paixao, E., Pereira, T., Iovita, R., Marreiros, J., 2019a. Back to the edge: relative coordinate system for use-wear analysis. Archael. Antropol. Sci. https://doi.org/10.1007/ s12520-019-00801-y.

Calandra, I., Schunk, L., Bob, K., Gneisinger, W., Pedergnana, A., Paixao, E., Hildebrandt, A., Marreiros, J., 2019b. The effect of numerical aperture on quantitative use-wear studies and its implication on reproducibility. Sci. Rep. 9, 1-10. https://doi.org/10.1038/s41598-019-42713-w.

Caux, S., Galland, A., Queffelec, A., Bordes, J.-G., 2018. Aspects and characterization of chert alteration in an archaeological context: a qualitative to quantitative pilot study. J. Archaeol. Sci.: Report 20, 210-219. https://doi.org/10.1016/j. jasrep.2018.04.027.

Clemente-Conte, I., Gibaja-Bao, J.F., 2009. Formation of use-wear traces in non-flint rocks: the case of quartzite and rhyolite - differences and similarities. In: Non-Flint Raw Material Use in Prehistory: Old Prejudices and New Directions/L'utilisation Préhistorique de Matière Premieres Lithiques Alternatives: Anciens Préjugés, Nouvelles Perspectives. BAR International Series 1939. Archeopress, Oxford, pp. 93-98.

Cotterell, B., Kamminga, J., 1987. The formation of flakes. Am. Antiq. 52, 675-708.

Evans, A.A., Donahue, R.E., 2005. The elemental chemistry of lithic microwear: an experiment. J. Archaeol. Sci. 32, 1733-1740. https://doi.org/10.1016/j. jas.2005.06.010.

Evans, A.A., MacDonald, D., 2011. Using metrology in early prehistoric stone tool research: further work and a brief instrument comparison. Scanning 33, 294-303. https://doi.org/10.1002/sca.20272.

Galland, A., Queffelec, A., Caux, S., Bordes, J.-G., 2019. Quantifying lithic surface alterations using confocal microscopy and its relevance for exploring the Châtelperronian at La Roche-à-Pierrot (Saint-Césaire, France). J. Archaeol. Sci. 104, 45-55. https://doi.org/10.1016/j.jas.2019.01.009.

Hamon, C., Plisson, H., 2008. Functional analysis of grinding stones: the blind-test contribution. In: Prehistoric Technology 40 Years Later : Functional Studies and the Russian Legacy, pp. 29-38.

Hoffman, M.D., Gelman, A., 2014. The No-U-turn sampler: adaptively setting path lengths in Hamiltonian Monte Carlo. J. Mach. Learn. Res. 15, 1593-1623.

Højsgaard, S., Halekoh, U., 2020. doBy: Groupwise Statistics, LSmeans, Linear Contrasts. Utilities. R package version 4.6.5 [WWW Document]. https://CRAN.R-project.org/ package $=$ doBy. (Accessed 21 January 2020).

Hughes, R.E., Högberg, A., Olausson, D., 2012. The chemical composition of some archaeologically significant flint from Denmark and Sweden. Archaeometry 54, 779-795. https://doi.org/10.1111/j.1475-4754.2011.00655.x.

Ibáñez, J.J., González-Urquijo, J.E., Gibaja, J., 2014. Discriminating wild vs domestic cereal harvesting micropolish through laser confocal microscopy. J. Archaeol. Sci. 48, 96-103. https://doi.org/10.1016/j.jas.2013.10.012.

Ibáñez, J.J., Lazuen, T., González-Urquijo, J., 2018. Identifying experimental tool use through confocal microscopy. J. Archaeol. Method Theor 26, 1176-1215. https:// doi.org/10.1007/s10816-018-9408-9.

International Organization for Standardization, 2012. ISO 25178-2 - Geometrical Product Specifications (GPS) - Surface Texture: Areal - Part 2: Terms, Definitions and Surface Texture Parameters.

James, D., Hornik, K., 2020. Chron: Chronological Objects which Can Handle Dates and Times. R package version 2.3-55 [WWW Document]. https://CRAN.R-project.org/p ackage $=$ chron. (Accessed 21 January 2020).

Kamminga, J., 1979. The nature of use-polish and abrasive smoothing on stone tools. In: Hayden, B. (Ed.), Lithic Use-Wear Analysis. Academic Press, New York, pp. 143-157.

Keeley, L.H., 1980. Experimental Determination of Stone Tool Uses.

Kruschke, J.K., 2013. Bayesian estimation supersedes the t test. J. Exp. Psychol. Gen. 142, 573-603. https://doi.org/10.1037/a0029146.

Levi Sala, I., 1986. Use wear and post-depositional surface modification: a word of caution. J. Archaeol. Sci. https://doi.org/10.1016/0305-4403(86)90061-0.

Macdonald, D.A., Evans, A.A., 2014. Evaluating surface cleaning techniques of stone tools using laser scanning confocal microscopy. Micros. Today 22, 22-27. https:// doi.org/10.1017/S1551929514000364. 
Macdonald, D.A., Stemp, W.J., Evans, A.A., 2018. Exploring the microscale: advances and novel applications of microscopy for archaeological materials. J. Archaeol. Sci.: Report. https://doi.org/10.1016/j.jasrep.2018.02.036, 0-1.

Mansur-Franchomme, M.E., 1983. Scanning electron microscopy of dry hide working tools: the role of abrasives and humidity in microwear polish formation. J. Archaeol. Sci. 10, 223-230.

McPherron, S.P., Braun, D.R., Dogandžić, T., Archer, W., Desta, D., Lin, S.C., 2014. An experimental assessment of the influences on edge damage to lithic artifacts: a consideration of edge angle, substrate grain size, raw material properties, and exposed face. J. Archaeol. Sci. 49, 70-82. https://doi.org/10.1016/j. jas.2014.04.003.

Pedergnana, A., 2017. Microwear and residue analyses of quartzite stone tools. Experimental development of a method and its application to the assemblages from the Pleistocene sites of Gran Dolina-TD10 (Sierra de Atapuerca, Burgos, Spain) and Payre (Ardèche, France) (PhD Thesis). Universitat Rovira i Virgili.

Pedergnana, A., Ollé, A., 2017. Monitoring and interpreting the use-wear formation processes on quartzite flakes through sequential experiments. Quat. Int. 427, 35-65. https://doi.org/10.1016/j.quaint.2016.01.053.

Pedergnana, Antonella, Asryan, L., Fernández-Marchena, J.L., Ollé, A., 2016. Modern contaminants affecting microscopic residue analysis on stone tools: a word of caution. Micron 86, 1-21. https://doi.org/10.1016/j.micron.2016.04.003.

Rots, V., 2010. Prehension and Hafting Traces on Flint Tools: a Methodology. Leuven University Press, Leuven.

Salvatier, J., Wiecki, T.V., Fonnesbeck, C., 2016. Probabilistic programming in Python using PyMC3 (No. e1686v1). PeerJ Inc. https://doi.org/10.7287/peerj. preprints.1686v1.
Schauberger, P., Walker, A., 2019. Openxlsx: Read, Write and Edit xlsx Files. R package version 4.1.4 [WWW Document]. https://CRAN.R-project.org/package=openxlsx. (Accessed 21 January 2020).

Scott, R.S., Ungar, P.S., Bergstrom, T.S., Brown, C.A., Childs, B.E., Teaford, M.F., Walker, A., 2006. Dental microwear texture analysis: technical considerations. J. Hum. Evol. 51, 339-349. https://doi.org/10.1016/j.jhevol.2006.04.006.

Stemp, W.J., Lerner, H.J., Kristant, E.H., 2013. Quantifying microwear on experimental mistassini quartzite scrapers: preliminary results of exploratory research using LSCM and scale-sensitive fractal analysis. Scanning 35, 28-39. https://doi.org/10.1002/ sca. 21032.

Vietti, L.A., 2016. Quantifying bone weathering stages using the average roughness parameter Ra measured from 3D data. Surf. Topogr. Metrol. Prop. 4, 034006 https:// doi.org/10.1088/2051-672X/4/3/034006.

Werner, J.J., 2018. An experimental investigation of the effects of post-depositional damage on current quantitative use-wear methods. J. Archaeol. Sci.: Report 17, 597-604. https://doi.org/10.1016/j.jasrep.2017.12.008.

Wickham, H., 2016. ggplot2: Elegant Graphics for Data Analysis. Springer, New York, USA.

Xie, Y., 2014. knitr: a comprehensive tool for reproducible research in R. In: Stodden, V., Leisch, F., Peng, R.D. (Eds.), Implementing Reproducible Computational Research. Chapman and Hall/CRC, Boca Raton, Florida.

Xie, Y., 2015. Dynamic Documents with R and knitr, second ed. Chapman and Hall/CRC, Boca Raton, Florida.

Xie, Y., Allaire, J.J., Grolemund, R., 2018. R Markdown: the Definitive Guide. Chapman \& Hall/CRC.

Yu, S., Oguchi, T., 2009. Complex relationships between salt type and rock properties in a durability experiment of multiple salt-rock treatments. Earth Surf. Process. Landforms 34, 2096-2110. 